\title{
Ferrocenliganden in der Lanthanid-Chemie: Chelatstabilisierung des ersten Diorganyllanthanid(III)-halogenids
}

Heinz Gornitzka, Alexander Steiner, Dietmar Stalke, Ulrike Kilimann, Frank T. Edelmann

Institut für Anorganische Chemie der Universität Göttingen, Tammannstr. 4, W-3400 Göttingen (Deutschland)

Klaus Jacob und Karl-Heinz Thiele

Institut für Anorganische Chemie der Technischen Universität Messeburg, Geusaer Straße, $0-4200$ Merseburg (Deutschland)

(Eingegangen den 25. Mai 1992)

Abstract

Dark red crystalline $\left[(\mathrm{FcN})_{2} \mathrm{Yb}(\mu-\mathrm{Cl})_{2} \mathrm{Li}(\mathrm{THF})_{2}\right](2)$ has been prepared by treatment of anhydrous $\mathrm{YbCl}_{3}$ with 2-(dimethylaminomethyl)ferrocenyllithium in a molar ratio of $1: 2$. 2 represents the first example of a diorganolanthanide(III)halide containing $\sigma$-bonded organic ligands. The molecular structure of 2 has been determined by an X-ray diffraction study.

Homoleptische Lanthanid(III)alkyle sind notorisch instabil [1]. Aufgrund der ausgeprägten Tendenz zur Ausbildung höherer Koordinationszahlen sind beispielsweise einfache Trimethyl- oder Triphenyllanthanid(III)-Verbindungen nicht isolierbar. Drei verschiedene Synthesestrategien führten dennoch zur Darstellung einiger stabiler Vertreter dieser Substanzklasse. Durch Einführung des sterisch anspruchsvollen Bis(trimethylsilyl)methyl-Substituenten gelang Lappert et al. die Synthese der homoleptischen, dreifach koordinierten Lanthanid(III)alkylverbindungen $\left[\mathrm{Ln}\left(\mathrm{CH}\left(\mathrm{SiMe}_{3}\right)_{2}\right\}_{3}\right]$ [2]. Manzer et al. erreichten eine kinetische Stabilisierung von Triaryllanthanid(III)-Komplexen mit Hilfe des chelatisierenden $\sigma$-Arylliganden 2-(Dimethylaminomethyl)phenyl [3]. Durch Bildung von at-Komplexen des Typs $\left[\mathrm{LnMe}_{6}\right]^{3-}$ gelangten schließlich Schumann $e t$ al. zu stabilen Lanthanid(III)-Verbindungen mit einfachen Alkylliganden [4]:

Correspondence to: Dr. F.T. Edelmann. 
$\left.\left[\mathrm{LniCH}\left(\mathrm{SiMe}_{3}\right)_{2}\right)_{3}\right]$

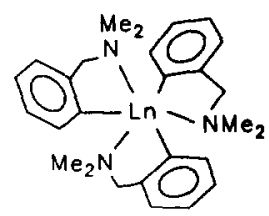

$\left[\mathrm{LnMe}_{6}\right]^{3-}$

Funktionalisierbare Dialkyl- oder Diaryllanthanid(III)halogenide konnten bislang nicht erhalten werden. Eine große Rolle spielen derartige Verbindungen allerdings in der Cyclopentadienylchemie der dreiwertigen Lanthanide. Hier zählen Komplexe des Typs $\left[\mathrm{Cp}_{2} \mathrm{LnCl}\right]_{2},\left[\mathrm{Cp}_{2}^{*} \mathrm{LnCl}(\mathrm{THF})\right]$ oder $\left[\mathrm{Cp}_{2}^{*} \operatorname{Ln}(\mu-\mathrm{Cl})_{2} \mathrm{Li}(\mathrm{THF})_{2}\right]$ zu den wichtigsten Ausgangsmaterialien [1]. Unter Verwendung des zur Chelatbildung befähigten und sterisch anspruchsvollen Liganden 2-(Dimethylaminomethyl) ferrocenyl $(=\mathrm{FcN})[5]$ gelang uns jetzt die Synthese des ersten Diorganyllanthanid (III)halogenids mit $\sigma$-gebundenen Organylliganden.

Wasserfreies Ytterbiumtrichlorid reagiert mit zwei Äquivalenten 2-(Dimethylaminomethyl)ferrocenyllithium (1) in THF unter Bildung einer roten Lösung, aus der sich nach Kristallisation aus Toluol dunkelrote Kristalle der Zusammensetzung $\left[(\mathrm{FcN})_{2} \mathrm{Yb}(\mu-\mathrm{Cl})_{2} \mathrm{Li}(\mathrm{THF})_{2}\right](2)$ isolieren lassen:

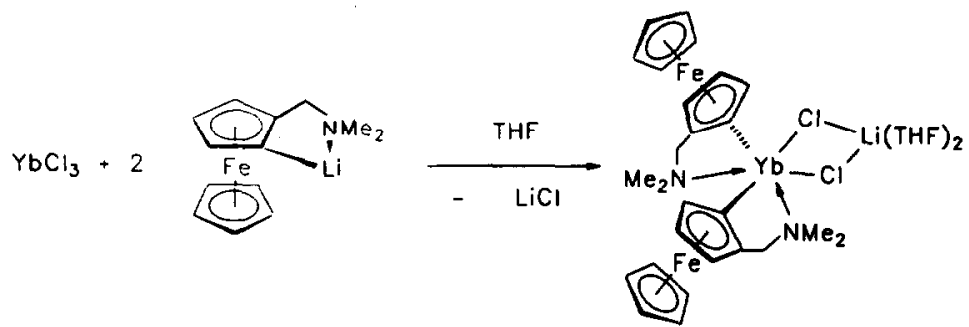

Trotz des koordinierten Lithiumchlorids ist 2 gut löslich in Toluol. Kristallines 2 ist äußerst luftempfindlich, aber thermisch sehr stabil. Die Bildung eines Diorganyllanthanid(III)halogenids wird dadurch ermöglicht, daß die Raumerfüllung des $\mathrm{FcN}$-Chelatliganden an die des Pentamethylcyclopentadienyls $\left(\mathrm{Cp}^{*}\right)$ heranreicht. Ebenso wie Komplexe des Typs $\left[\mathrm{Cp}_{3}^{\star} \mathrm{Ln}\right.$ ] auf konventionellem Wege nicht zugänglich sind [6], reagiert $\mathrm{YbCl}_{3}$ aus sterischen Gründen auch nicht mit drei Äquivalenten 1 zum homoleptischen Derivat $\left[(\mathrm{FcN})_{3} \mathrm{Yb}\right]$.

Die Tieftemperatur-Röntgenstrukturanalyse $\left[7^{*}, 8^{*}\right]$ bestätigt das Vorliegen des ersten Diorganyllanthanid(III)halogenids (Fig. 1).

Durch Koordination von Lithiumchlorid erreicht das Ytterbium die formale Koordinationszahl 6. Die Koordinationssphäre am Lithium wird durch zwei THFLiganden vervollständigt. Komplexe mit einem zentralen viergliedrigen $\operatorname{Ln}(\mu$ $\mathrm{Cl}_{2}$ Li-Ring waren bis vor kurzem nur von den Cyclopentadienyl-Derivaten

\footnotetext{
* Die Literaturnummer mit einem Sternchen deutet eine Bemerkung in der Literaturliste an.
} 


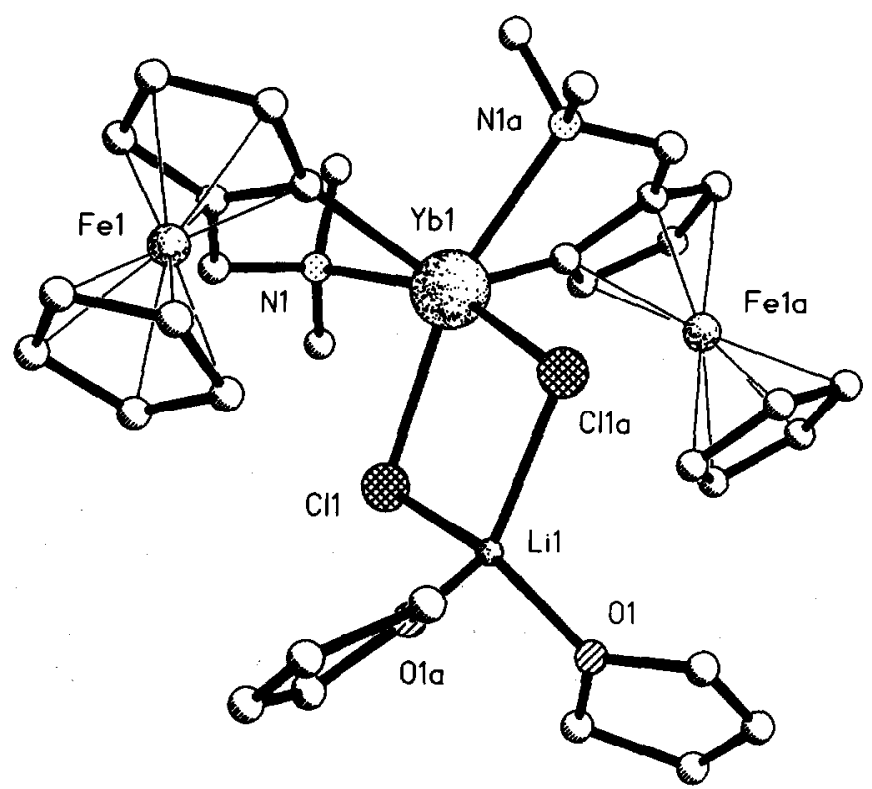

Fig. 1. Molekülstruktur von 2 im Kristall [7,8]. Ausgewählte Bindungslängen (pm) und -winkel ( ${ }^{\circ}$ : $\mathrm{Yb}(1)-\mathrm{C}(1)$ 265.1(2), $\mathrm{Yb}(1)-\mathrm{N}(1)$ 255.5(6), $\mathrm{Yb}(1)-\mathrm{C}(14)$ 237.1(7), Fe(1)-C(14) 210.9(7), Cl(1)-Li(1) 236.4(14), N(1)-C(20) 148.0(9), C(10)-C(20) 149.8(10), C(10)-C(14) 145.2(9), Cl(1)-Yb(1)-C(14) 108.6(2), N(1)-Yb(1)-C(14) 71.4(2), Cl(1)-Yb(1)-Cl(1a) 80.9(1), N(1)-Yb(1)-Cl(1a) 161.3(1), C(14)$\mathrm{Yb}(1)-\mathrm{C}(14 \mathrm{a})$ 108.1(2), N(1)-Yb(1)-C(14a) 83.3(2), C(14)-Yb(1)-C(14a) 131.1(3), Cl(1)-Li(1)-Cl(1a) 93.4(7), $\mathrm{Cl}(1)-\mathrm{Yb}(1)-\mathrm{N}(1)$ 81.6(1), $\mathrm{Cl}(1)-\mathrm{Yb}(1)-\mathrm{N}(1 \mathrm{a})$ 116.4(3), $\mathrm{C}(14)-\mathrm{Yb}(1)-\mathrm{N}(1 \mathrm{a})$ 83.3(2), $\mathrm{Yb}(1)-$ Cl(1)-Li(1) 92.8(4).

$\left[\mathrm{Cp}_{2}^{\star} \operatorname{Ln}(\mu-\mathrm{Cl})_{2} \mathrm{Li}(\mathrm{L})_{2}\right]\left(\mathrm{L}=\mathrm{THF}, 1 / 2 \mathrm{DME}, \mathrm{Et}_{2} \mathrm{O}\right)$ bekannt [1]. Neuere Untersuchungen haben jedoch gezeigt, daß dieser Verbindungstyp weiter verbreitet ist und beispielsweise auch bei Lanthanidkomplexen mit sterisch anspruchsvollen Chelatliganden auftritt $[9,10]$.

Bei Raumtemperatur verwittern die tiefroten Einkristalle von 2 sehr leicht, da zusätzlich zum koordinativ gebundenen THF noch weitere THF-Moleküle in die Kristallstruktur eingebaut sind. Fig. 2 macht deutlich, daß sich die Moleküle von 2 stapelförmig anordnen. Zwischen diesen Stapeln bilden sich Kanäle, in die Kristall-THF eingelagert werden kann.

\section{Arbeitsvorschrift}

Die Darstellung von $2 \mathrm{muB}$ unter sorgfältigem Luftausschluß erfolgen.

2: Zum Gemisch aus $1.96 \mathrm{~g}(7.0 \mathrm{mmol}) \mathrm{YbCl}_{3}$ und $3.94 \mathrm{~g}(14.0 \mathrm{mmol}) \mathrm{Li}(\mathrm{FcN})$ [5] gibt man $100 \mathrm{ml}$ THF. Nach $24 \mathrm{~h}$ Rühren bei Raumtemperatur wird der Rückstand in $100 \mathrm{ml}$ Toluol aufgenommen und vom ausgefallenen $\mathrm{LiCl}$ abfiltriert. Man engt das Filtrat auf ca. $20 \mathrm{ml}$ ein und versetzt mit $20 \mathrm{ml}$ THF. Abkühlen auf $-20^{\circ} \mathrm{C}$ liefert $0.60 \mathrm{~g}(0.7 \mathrm{mmol}, 10 \%)$ große, dunkelrote Einkristalle vom $\mathrm{Fp}$. $106^{\circ} \mathrm{C}$. Durch Eindampfen der Mutterlauge und Waschen mit Hexan läßt sich weiteres, mikrokristallines Material gewinnen. Gef.: C, 45.9; H, 5.3; N, 3.2. $\mathrm{C}_{34} \mathrm{H}_{48} \mathrm{Cl}_{2} \mathrm{Fe}_{2} \mathrm{LiN}_{2} \mathrm{O}_{2} \mathrm{Yb}(879.3)$ ber.: $\mathrm{C}, 46.4 ; \mathrm{H}, 5.5 ; \mathrm{N}, 3.2 \%$. IR (Nujol): $1262 \mathrm{~m}$, $1104 \mathrm{~m}, 1049 \mathrm{~m}, 1025 \mathrm{~s}, 996 \mathrm{~m}, 801 \mathrm{~m}, 722$ vs $\mathrm{cm}^{-1}$. MS: $m / z 371$ $\left(\mathrm{Yb}\left(\mathrm{C}_{5} \mathrm{H}_{3}\left(\mathrm{CH}_{2} \mathrm{NMe}_{2}\right)\right)\left(\mathrm{C}_{5} \mathrm{H}_{3}\left(\mathrm{CH}_{2}\right)\right), 2 \%\right), 273\left(\mathrm{Fc}\left(\mathrm{CH}_{2} \mathrm{NMe}_{2}\right), 96\right), 199\left(\mathrm{FcCH}_{2}\right.$, 

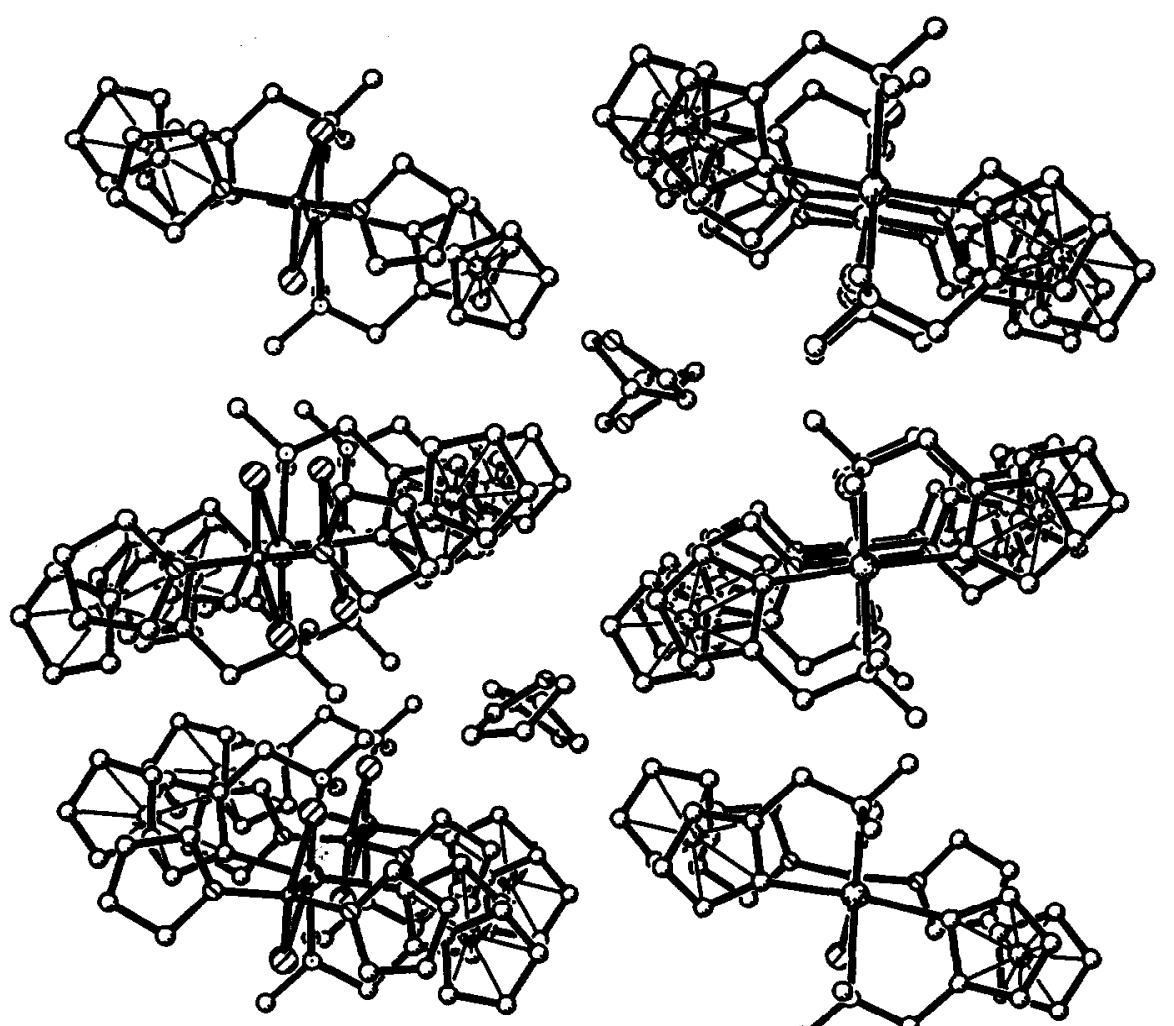

Fig. 2. Kristallstruktur von 2.

$100), 186\left(\mathrm{Cp}_{2} \mathrm{Fe}, 16\right), 121(\mathrm{CpFe}, 38), 56(\mathrm{Fe}, 10) .{ }^{1} \mathrm{H}-\mathrm{NMR}\left(250 \mathrm{MHz}, \mathrm{C}_{6} \mathrm{D}_{6}\right): \delta$ $55.2(\mathrm{~m}, 8 \mathrm{H}, \mathrm{THF}), 42.7$ (m, 8H, THF), $-18.4\left(\mathrm{br}, 12 \mathrm{H}, \mathrm{NMe}_{2}\right.$ ), -29.7 (br, $10 \mathrm{H}$, $\left.\mathrm{C}_{5} \mathrm{H}_{5}\right),-32.4\left(\mathrm{~m}, 4 \mathrm{H}, \mathrm{CH}_{2}\right),-34.3\left(\mathrm{~m}, 6 \mathrm{H}, \mathrm{C}_{5} \mathrm{H}_{3}\right) \mathrm{ppm}$.

Dank. Für finanzielle Unterstützung danken wir der Deutschen Forschungsgemeinschaft und der Dr. Otto Röhm-Gedächtnisstiftung.

\section{Literatur und Bemerkungen}

1 H. Schumann, Angew. Chem., 96 (1984) 475; Angew. Chem., Int. Ed. Engl., 23 (1984) 474.

2 P.B. Hitchcock, M.F. Lappert, R.G. Smith, R.A. Bartlett und P.P. Power, J. Chem. Soc., Chem. Commun., (1988) 1007.

3 L.E. Manzer, J. Am. Chem. Soc., 100 (1978) 8068.

4 H. Schumann, J. Müller, N. Bruncks, H. Lauke, J. Pickardt, H. Schwarz und K. Eckardt, Organometallics, 3 (1984) 69.

5 M.D. Rausch, G.A. Moser und C.F. Meade, J. Organomet. Chem., 51 (1973) 1.

6 W.J. Evans, S.L. Gonzales und J.W. Ziller, J. Am. Chem. Soc., 113 (1991) 7423.

$72\left(M_{\mathrm{r}}=991.3\right)$ : Orthorhombisch, Raumgruppe $P b c n, a=1547.7(4), b=1133.9(2), c=2515.1(4) \mathrm{pm}$, $V=4.4140 \mathrm{~nm}^{3}, Z=4, D_{\text {ber. }}=1.492 \mathrm{~g} \mathrm{~cm}^{-3}$, KristallgröBe $0.5 \times 0.4 \times 0.3 \mathrm{~mm}^{3}$, Siemens-Stoe AED Vierkreisdiffraktometer, $2 \theta_{\max }=47^{\circ}, 3251$ symmetrieunabhängige Reflexe, davon 2462 mit $F>3 \sigma F$ fïr die Verfeinerung verwendet (shelXTL PLus), Meßtemperatur $T=-120^{\circ} \mathrm{C} ; 255$ verfeinerte Parameter, $R=0.049, R_{\mathrm{w}}=0.051, w^{-1}=\sigma^{2}(F)+0.0005 F^{2}$. 
8 Weitere Einzelheiten zur Kristallstrukturanalyse können beim Fachinformationszentrum Karlsruhe, Gesellschaft für wissenschaftlich-technische Information mbH, W-7514 Eggenstein-Leopoldshafen 2, unter Angabe der Hinterlegungsnummer CSD-56247, der Autoren und des Zeitschriftenzitats angefordert werden.

9 A. Recknagel, A. Steiner, S. Brooker, D. Stalke und F.T. Edelmann, J. Organomet. Chem., 415 (1991) 315.

10 A. Recknagel, F. Knösel, H. Gornitzka, M. Noltemeyer, F.T. Edelmann und U. Behrens, J. Organomet. Chem., 417 (1991) 363. 